\title{
Erinnerungsworte an Paul Ehrlich anläßlich seines hundertsten Geburtstages
}

\author{
Von P. KarRer, Zürich
}

Der 14. März dieses Jahres war der hundertste Geburtstag Paul Ehrlichs. Dieser Umstand bildete den Anlaß, daß das Andenken dieses großen Forschers und Gelehrten in verschiedenen Ländern, vorab in Deutschland, erneuert und der hundertste Geburtstag festlich begangen wurde. Die Schweizerische Naturforschende Gesellschaft war der Meinung, daß auch an ihrer Jahrestagung Worte des Gedenkens erklingen sollten, und ersuchte mich, als ehemaligen Mitarbeiter Paul Ehrlichs, dessen Persönlichkeit und Lebenswerk in einer kurzen Ansprache wieder aufleben zu lassen.

Wenn man die Persönlichkeit eines Forschers genauer kennenlernen will, ist es oft zweckmäßig, seine Arbeitsstätte aufzusuchen und sich seine tägliche Umgebung näher anzuschauen. Wie sah diese bei Paul Ehrlich aus? 1899 hatte Ehrlich im Alter von fünfundvierzig Jahren die Direktion des königlichen Institutes für experimentelle Therapie in Frankfurt am Main übernommen, und 1906 wurde ihm auch die Leitung des neugegründeten Georg-Speyer-Hauses übertragen, das, eine private Stiftung, chemotherapeutischer Forschung dienen sollte. In diesen beiden Forschungsstätten hat er bis zu seinem frühen Tod im Jahre 1915 gewirkt; es war der erfolgreichste und entscheidende Abschnitt seines Lebens.

Seine eigenen Arbeitsräume lagen im Institut für experimentelle Therapie. Sie bestanden aus zwei Zimmern, einem etwa $20 \mathrm{~m}^{2}$ großen chemischen Laboratorium und einem anstoßenden, etwa halb so großen Schreibzimmer. In der Mitte des Laboratoriums stand ein großer chemischer Experimentiertisch mit Wasser- und Gasanschlüssen. Dieser war bis an den äußersten Rand mit Flaschen und Fläschchen besetzt, in denen sich Hunderte von verschiedenen Substanzen befanden. Zum Arbeiten war daher auf dem Tisch eigentlich kein Platz; man konnte sich lediglich der beiden Bunsenbrenner bedienen; diese waren denn auch die eigentlichen Zentren des Arbeitsraumes; hier hat Paul Ehrlich oft während Stunden Reagenzglasversuche ausgeführt, nach Färbungen und Farbstoffen gesucht und, rein empirisch, manche erstaunliche Beobachtung gemacht.

Das anstoßende Schreibzimmer enthielt ein kleines Schreibpult und ein bescheidenes Sofa. Aber beide waren schon lange ihren eigentlichen Bestimmungen entzogen, denn sie waren mit Büchern, Zeitschriften und Se- 
paraten belegt, die sich fast bis an die Decke hinauftürmten. Da also hier nicht mehr geschrieben und noch viel weniger geruht werden konnte, mußte zum Unterzeichnen der Briefe ein kleiner Schreibtisch benutzt werden, welcher im Laboratorium stand, der aber auch nur so viel freie Tischfläche aufwies, daß man gerade noch einen Brief hinlegen konnte. Es kam auch vor, daß selbst dieser letzte Rest von Tischfläche versperrt war; dann hat man wohl zwei Sägeblöcke im Laboratorium aufgestellt und ein ungehobeltes Brett darüber gelegt, auf dem dann die Unterschriften gegeben wurden.

Bequeme Stühle waren selbstverständlich keine da, sondern nur zwei kleine gewöhnliche Holzstühle. Besucher - und es kamen viele und bedeutende Männer - mußten mit dieser primitiven Sitzgelegenheit vorlieb nehmen, wenn sie nicht, was meistens der Fall war, stehend empfangen wurden.

Paul Ehrlich betrat sein Institut meistens gegen zehn Uhr. Dann erkundigte er sich zuerst über die Arbeiten der verschiedenen Abteilungen, machte einen Rundgang durch alle Laboratorien, sprach mit seinen Mitarbeitern; zwischendurch diktierte er stehend Briefe oder entwarf wenigstens einige Gedanken, welche seine Sekretärin dann in Briefform brachte. Inzwischen war es ein Uhr geworden; hierauf trank er, ohne die Arbeit zu unterbrechen, eine Tasse Suppe. Der Nachmittag war dann sehr oft die Zeit, da er vor dem Laboratoriumstisch stand und einen Reagenzglasversuch nach dem andern ausführte. Und wenn er glaubte, eine bemerkenswerte Beobachtung gemacht zu haben, wurde sie sofort zu Papier gebracht und einem Mitarbeiter geschickt, damit sie dieser näher studiere. Ich erhielt so oft zwei, drei ja vier gelbe Karten pro Tag, auf denen er mich bat, dieses oder jenes genauer zu untersuchen. - Abends fünf Uhr oder halb sechs Uhr wurde der rastlose Mann, für den die Zeit nicht zu existieren schien, von seiner Gattin telephonisch zum Abendessen gerufen. Den langen Abend verbrachte er dann, in Rauchwolken gehüllt, in seiner großen Bibliothek, wo er las, wissenschaftliche Arbeiten schrieb, vor allem aber seiner Phantasie freien Lauf ließ, die ihm so häufig glückliche und bedeutsame Einfälle vermittelte.

Ich habe Ihnen ein etwas eingehenderes Bild von der Lebensweise Paul Ehrlichs vermittelt, weil aus ihm hervorgeht, daß bei diesem großen Gelehrten und unendlich bescheidenen Menschen Äußerlichkeiten und Komfort keine Rolle spielten, ebensowenig wie Reichtum, den er nur für die Wissenschaft einsetzte. Er war ein Mann, der mit jeder Faser der Wissenschaft verfallen war, der sich nur für die Forschung interessierte und sich auch für diese aufopferte. 
Paul Ehrlich wurde am 14.März 1854 in Strehlen in Schlesien geboren. Er studierte Medizin in Breslau, Straßburg, Freiburg und Leipzig. Zu seinen Lehrern gehören Waldeyer, Heidenhain, Cohnheim, ferner Carl WeiGert. Später arbeitete er als Assistent bei Frerichs und Gerhardt, vor allem aber von 1890-1896 bei Roвert Косн.

Schon als Student der Medizin hat er eine große Zahl von wissenschaftlichen Abhandlungen publiziert, die sich auf völlig neuen Bahnen bewegten. Charakteristisch für die Art, wie er aus scheinbar unbedeutenden Beobachtungen wichtige Anregungen und Schlußfolgerungen ziehen konnte, ist eine Episode, welche in seine Studienjahre fiel. Er hatte eine Arbeit von HevBel über Bleivergiftung gelesen, in der behauptet wurde, daß die Zellen derjenigen Organe, in denen sich bei Bleivergiftung dieses Metall findet, auch in totem Zustande Blei aus seinen Lösungen im Zellinnern abzuscheiden imstande sind. Ehrlich schloß daraus, daß diese Zellen ein selektives Verankerungsvermögen für Blei besitzen müssen, und er ging sofort noch einen Schritt weiter, indem er sich sagte, daß wahrscheinlich nicht nur das Blei, sondern auch manche andere Stoffe eine solche selektive Verwandtschaft zu bestimmten Zellen des Organismus aufweisen werden. Er prüfte diese Frage mit Farbstoffen und fand seine Vermutung bestätigt. Dies war der unscheinbare Anstoß zu seinen nun folgenden, grundlegenden Arbeiten über die selektiven Färbungen der Zellen, die Vitalfärbungen, die einen gewissen Höhepunkt in der Auffindung einer selektiven Färbung der Tuberkelbazillen erreichten. Das Arbeiten mit Farbstoffen in einem medizinischen Laboratorium war für die damalige Zeit etwas Ungewöhnliches, und es ist begreiflich, daß die Kliniker von der Begleiterscheinung des Färbens, der Beschmierung der Tische durch Farbstoffspuren, nicht sehr begeistert waren. Ein solcher älterer Kollege hat daher einmal dem Studenten Paul Ehrlich vorgehalten: «Überall wo Sie arbeiten, hinterlassen Sie Spuren», ein Ausspruch, an den der gereifte Meister später mit Vergnügen zurückdachte.

So verschiedenartig die Forschungsgebiete Paul Ehrlichs scheinen mögen, werden sie doch von einem gemeinsamen Gedanken beherrscht und verbunden, daß die biologische Wirkung aller Stoffe konstitutionsspezifisch ist, daß die Struktur einer wirksamen chemischen Verbindung genau auf die Bestandteile der Zelle abgestimmt sein muß und daß eine biologische Wirkung eine Verankerung der chemischen Substanz an der Zelle voraussetzt. "Corpora non agunt nisi fixata» - dieses Postulat stellte er dem in der älteren Chemie geprägten Wort «corpora non agunt nisi soluta» gegenüber. 
In seinen Zell- und Vitalfärbungen, in seinen Arbeiten über Antigene und Antikörper, in seinen chemotherapeutischen Forschungen schien er diese seine Theorie bestätigt zu finden. So sagt er einmal in der anschaulichen Sprache, deren er sich zu bedienen pflegte:

«Wenn wir mit einer bestimmten Körperklasse, z. B. den Arsenikalien, bei verschiedenen Infektionskrankheiten eine sozusagen spezifische Therapie treiben wollen, so ist das nur in der Weise möglich, daß wir für jeden Parasiten bestimmte verankernde und ihm gewissermaßen eigenartige „Nebengruppierungen' auffindig machen, die bestimmte Reste packen und so eine spezifische Verankerung ermöglichen.» Und ein anderes Mal prägte er den klassisch gewordenen Ausspruch: «Wir müssen also zielen lernen, und zielen lernen durch chemische Variation.»

Paul Ehrlichs jüngere Forscherjahre waren erfüllt von seinen Arbeiten über die Zellfärbungen, Vitalfärbungen, denen sich grundlegende Untersuchungen über die Histologie und Physiologie der Blutzellen anschlossen. Es gelang ihm, mit Hilfe von Farbstoffen zu zeigen, daß zwei Arten von weißen Blutkörperchen existieren, Lymphozyten und Leukozyten, und er konnte auch die Elemente, aus denen sie sich aufbauen, scharf differenzieren. Noch größer waren die theoretischen und praktischen Erfolge, als er begann, sich mit den Problemen der Immunitätslehre zu beschäftigen. Hier schuf er Methoden, um Tiere hochgradig zu immunisieren, und nachher solche, um die Antikörper quantitativ zu messen und die Sera zu standardisieren; damit hat er die praktische Anwendung der Heilsera erst ermöglicht.

Diese Arbeiten wurden wohl durch die Entdeckung des Diphtherietoxins durch Roux und Yersin und die Entdeckung der Antitoxine durch BeHRING angeregt. Aber Ehrlich ging seinen eigenen Weg. Im Gift des RicinusSamens, dem Ricin, fand er einen Stoff, welcher ähnlich wie Bakterientoxine im tierischen Organismus Antikörper erzeugte und sich andererseits durch seine Fähigkeit, rote Blutkörperchen zu verklumpen und in größerer Menge aufzulösen, leicht nachweisen ließ. Mit diesem handlichen Toxinmodell konnte er nun die quantitativen Beziehungen aufklären, die zwischen dem Toxin und dem Antitoxin bestehen. Er schuf damit die Grundlage für viele andere Untersuchungen über Bakteriengiftstoffe und die durch sie erzeugten Antikörper, die von ihm selbst, von BeHring und einer Reihe anderer Forscher im Institut von Roвert Kосн in Berlin ausgeführt wurden. Als sich dann die Notwendigkeit zeigte, zur Kontrolle der neu eingeführten Heilsera eine staatliche Kontrollstelle zu schaffen, wurde diese 1896 Paul Ehrlich übertragen; kurze Zeit befand sich das Institut in Berlin-Steglitz 
und von 1899 an in Frankfurt am Main. Hier fand der Forscher die Zeit und Gelegenheit, die Beziehungen zwischen Toxinen und Antitoxinen, insbesondere auch am Beispiel des Diphtherietoxins, in allen Einzelheiten zu erforschen, exakte Meßmethoden auszuarbeiten und die Toxinforschung und Serologie auf gesicherte Grundlagen zu stellen. Wie groß angelegt diese Arbeiten waren, läßt sich aus dem Umstand ermessen, daß zu ihrer Durchführung etwa 10000 Meerschweinchen dienten.

Die Probleme der Immunitätslehre führten Paul Ehrlich schließlich auch dazu, die sogenannte Seitenkettentheorie aufzustellen, die, von vielen begeistert aufgenommen und begrüßt, von anderen scharf abgelehnt, doch einen überwältigenden Einfluß auf das Denken und die Betrachtungsweise in der Immunitätslehre ausgeübt hat, so daß Ehrlich in einem Vortrag, den er 1908 in der Deutschen Dermatologischen Gesellschaft hielt, mit Recht sagen durfte: "Ich darf wohl für mich das Verdienst in Anspruch nehmen, daß ich als erster von der Notwendigkeit, die Therapie vom distributiven Standpunkte aus zu betreiben, mich leiten ließ. Diese Anschauung ist auch die Quelle der Seitenkettentheorie geworden, von der auch die Gegner mir zugestehen müssén, daß sie auf den Gang der modernen Immunitätsforschung einen sehr erheblichen Einfluß ausgeübt hat».

Diese Seitenkettentheorie stützt sich einmal auf die Anschauung, daß ein toxischer Stoff nur dann wirken kann, wenn er von besonderen haptophoren Gruppen der Zelle verankert wird. Dies trifft insbesondere auch zu für die Toxine. Die Seitenkettentheorie erklärt nun die Bildung der Antitoxine (Antikörper) in der Weise, daß das Toxin sich zunächst mit bestimmten Zellrezeptoren verbindet; diese werden dann unter der Reizwirkung des Toxins im Übermaß von der Zelle produziert, von dieser losgerissen und gelangen, immer noch mit der haptophoren Gruppe versehen, in die Blutbahn, wo sie das allenfalls vorhandene Toxin abzufangen und zu neutralisieren vermögen. In neuerer Zeit wurde durch zahlreiche Arbeiten nachgewiesen, daß Plasmazellen die Rolle von Antikörperbildnern ausüben.

Die Seitenkettentheorie ist zu Lebzeiten Ehrlichs namentlich von Metschnikoff und dessen Schule angefochten worden, der die Phagozyten als diejenigen Gebilde betrachtete, welche eingedrungene Bakterien und andere Zellen, die ebenfalls Abwehrfermente erzeugen, zerstören. Während die Makrophagen rote Blutkörperchen und andere Zellen fressen, räumen die Mikrophagen mit den Bakterien auf. Metschnikoff gab aber schließlich zu, daß seine Phagozytentheorie keinen unbedingten Gegensatz zur Ehrlichschen Seitenkettentheorie darstelle und sich mit dieser vereinen 
lasse. In den letzten vierzig Jahren seit dem Tode Ehrlichs sind über die Wechselwirkungen zwischen Antigenen und Antikörpern verschiedene andere Theorien aufgestellt worden, welche an Stelle der Ehrlichschen Seitenkettentheorie vorgeschlagen wurden. So hat PAUling folgende Vorstellungen entwickelt: Das Antigen zieht die freien Enden einer Globulin-Polypeptidkette an und formt diese in ganz bestimmter Weise zu einer spezifischen Struktur, die zur Antigenstruktur komplementär ist. Der übrige Teil der Globulinmolekel formt und faltet sich dagegen nach anderen, ihr innewohnenden Gesetzmäßigkeiten und reißt sich dabei vom Antigen los. Schließlich liegt ein frei beweglicher Antikörper vor, dessen Struktur einerseits durch den Eingriff des Antigens, andererseits durch die unabhängig davon erfolgte Faltung der Polypeptidkette bestimmt wurde. Diese Theorie nimmt also - und damit geht sie etwas über die Ehrlichsche Seitenkettentheorie hinaus - eine aktive Formung des Antikörpers durch das Antigen an. In den vom Antigen spezifisch geformten Molekülenden der Polypeptidkette erkennt man aber unschwer die haptophoren Gruppen der Ehrlichschen Terminologie.

Als ein weiterer Ersatz der Seitenkettentheorie wurde von Burnet und Fenner sowie von Savag eine sogenannte Fermenttheorie postuliert, die besagt, daß das Antigen Fermente, welche der Globulinproduktion dienen, spezifisch so verändert, daß sie einen neuen Dauerzustand annehmen, welcher spezifisch auf das Antigen abgestimmt ist. Diese Hypothese verlegt somit die spezifische Strukturformung durch das Antigen von einem Globulinmolekül auf ein Ferment. Die Idee der konstitutionsspezifischen Beziehung zwischen Antikörper und Antigen, die den Kernpunkt der Seitenkettentheorie bildet, ist auch in ihr enthalten. So sehen wir, daß fünfzig Jahre Forschung auf diesem Gebiet wohl gewisse Änderungen in den Auffassungen zeitigten, diese aber Strukturelemente enthalten, die der Schöpfer der Seitenkettentheorie in seiner bilderreichen Sprache auszudrücken versuchte, wobei man sich durchaus klar ist, daß auch die heutigen Theorien eines Tages wohl vertieft oder umgestaltet oder durch etwas Besseres ersetzt werden müssen. Ehrlich selbst hat gesagt, daß «ein Fortschritt in der Erkenntnis nur vom theoretischen Gesichtspunkt aus erfolgen kann und eine verfehlte Theorie immer noch frùchtbringender wirke als rohe Empirie, die ohne Erklärungsversuch die Tatsachen registriert». Dieser Ausspruch kann in seiner lapidaren Form vielleicht als etwas zu weitgehend empfunden werden, er ist aber charakteristisch für diesen Forscher, dessen Phantasie keine Grenzen kannte und dem seine sprudelnden neuen Ideen und 
Einfälle stets Anregungen zu neuen, streng wissenschaftlichen Forschungen geworden sind.

Auch das Krebsproblem hat Ehrlich interessiert. Es gelang ihm, die Virulenz von Mäusetumoren so zu steigern, daß sich diese mit Sicherheit auf andere Versuchstiere übertragen ließen. Erst dadurch wurden Versuche möglich, welche erkennen lassen, welche Eingriffe oder Bedingungen die Resistenz der Tiere gegen Tumoren erhöhen können. Ehrlich huldigte schon der Auffassung, daß die Ursache des Krebses eine parasitäre ist, vergleichbar den Bakterien; sie lebt gegenwärtig weiter in der Anschauung, daß der Erreger der Tumoren ein Virus sein könnte.

Das letzte große Arbeitsgebiet, dem sich Paul Ehrlich zuwandte, war die experimentelle Chemotherapie. Diese Forschungen brachten ihm die größten wissenschaftlichen und praktischen Erfolge und eine ungewöhnliche Popularität. Auch bei diesen Arbeiten ließ er sich von seiner Theorie leiten, daß man unter den Tausenden von chemischen Verbindungen solche suchen muß, welche sich mittels bestimmter Atomgruppen an den Zellen des Parasiten verankern können; die Lösung dieser Aufgabe ist aber dadurch erschwert, daß die wirksame chemische Substanz für den Parasitenträger, also z. B. den Menschen, wenig toxisch sein muß. «Tatsächlich können nur Stoffe», sagt Ehrlich in einem Vortrag über die Grundlagen der experimentellen Chemotherapie «als Heilmittel wirken, die so beschaffen sind, daß die Parasiten maximal, die Körperorgane minimal geschädigt werden ». Oder anders ausgedrückt: die Parasitotropie muß die Organotropie sehr stark übertreffen.

Die ersten chemischen Verbindungen, die unter Ehrlichs Leitung als Chemotherapeutika studiert wurden, waren Farbstoffe, solche der Benzidinreihe (Trypanrot, Trypanblau) und der Triphenylmethanreihe (z. B. Tryparosan, ein halogenisiertes Parafuchsin). Wenn sie auch an mit Trypanosomen infizierten Mäusen eine gewisse Heilwirkung aufwiesen, so waren sie doch relativ schwach wirksam. Erst die Arbeiten mit organischen Arsenverbindungen führten zu dem erhofften entscheidenden Fortschritt. RoBERT КосH hatte das 1863 von BÉCHAMP erstmals dargestellte Atoxyl mit einigem Erfolg bei der Schlafkrankheit angewandt, und P. UhLenhut hatte gezeigt, daß sich damit syphilitische Kaninchen heilen lassen. Als sich Ehrlich diesem Präparat zuwandte, klärte er zunächst seine Konstitution auf und verbesserte seine Eigenschaften durch die Darstellung verschiedener Derivate, wie des Arsacetins und Arsenophenylglycins. Aber erst nach jahrelangen weiteren Forschungen reifte im Salvarsan der große Erfolg her- 
an, der ein neues Zeitalter der Bekämpfung von Infektionskrankheiten eröffnete. Man kann sich heute kaum mehr eine richtige Vorstellung von dem großen Aufsehen und der großen Begeisterung machen, die durch die ganze Welt gingen, als nicht nur die Fachzeitschriften, sondern auch die ganze Tagespresse verkündigte: die Syphilis ist nicht mehr eine unheilbare Krankheit, die Trypanosomiasen der Tropen (Schlafkrankheit, Tsetse, Durine, Aleppobeule) können mit Erfolg bekämpft werden, Frambösie und Rückfallfieber lassen sich ausrotten, Rotz, Rotlauf und Malaria tertiana günstig beeinflussen.

Für Paul Ehrlich war der Erfolg kein Grund zum Stillestehen. Im Gegenteil, er strebte mit derselben Energie weiter. Noch waren die geschaffenen neuen Chemotherapeutika keine Idealmittel, sie besaßen noch eine nicht zu vernachlässigende Giftigkeit. Außerdem wurden manche Parasitenstämme unter ihrer Einwirkung gegen diese Verbindungen resistent. Diese Tatsachen veranlaßten Ehrlich, die Arzneifestigkeit der Parasiten in groß angelegten Untersuchungen zu studieren und nach neuen, noch wirksameren Chemotherapeutika zu suchen.

Heute ist der Begriff der Arzneifestigkeit Allgemeingut der Medizin und Biologie geworden. Wer aber denkt daran, daß er kaum eine halbes Jahrhundert alt ist und von Paul Ehrlich auf Grund tiefschürfender experimenteller Arbeiten erschlossen wurde? Bei der Prüfung von Arsenverbindungen auf trypanozide Wirkung machte er die Beobachtung, daß bei ungenügender Dosierung des Arsenikals Rezidive auftraten und die nun im Blut des Tieres wieder erschienenen Trypanosomen gegen das Heilmittel widerstandsfähiger geworden waren. Nach weiterer Behandlung der Krankheitserreger mit derselben Arsenverbindung stieg deren Resistenz immer mehr, bis sie schließlich auf das Heilmittel überhaupt nicht mehr ansprachen: sie waren «arsenfest» geworden. Diese Arzneifestigkeit blieb dem betreffenden Trypanosomenstamm erhalten, d. h. es war eine dauernde, vererbbare Änderung des Krankheitserregers erfolgt. Diese Erkenntnis war für die praktische Bekämpfung der Infektionskrankheiten von grundlegender Bedeutung. Ehrlich selbst hat den Weg angegeben, wie die Arzneifestigkeit vermieden werden soll; er nannte ihn "Therapia sterilisans magna» und wurde nicht müde, sich für diese Behandlungsmethode mit größtmöglichen Anfangsdosen des Chemotherapeutikums einzusetzen.

Paul Ehrlich hat die Arzneifestigkeit damit erklärt, daß im Mikroorganismus bestimmte "Chemozeptoren», d. h. spezifische Gruppen, welche das Chemotherapeutikum binden können, bei der Arzneifestigung so verändert 
werden, daß sie weniger und schließlich gar keine Affinität mehr zum chemotherapeutischen Stoff zeigen. Er erkannte auch, daß die erworbene Arzneifestigkeit eines Mikroorganismus gegenüber Chemotherapeutika weitgehend konstitutionsspezifisch, d. h. an die Konstitution des betreffenden Stoffes gebunden, ist. Allerdings gibt es Ausnahmen: Ehrlich selbst konnte zeigen, daß arsenfeste Trypanosomenstämme auch gegen bestimmte Acridinfarbstoffe (und einige verwandte Pigmente) unempfindlich geworden waren.

Nach Ehrlichs 1915 erfolgten Tode wurde es zunächst auf dem Gebiet der Chemotherapie still; dann setzten systematische chemotherapeutische Untersuchungen, vornehmlich in Fabriklaboratorien, ein. Sie führten allmählich zu großen Erfolgen. Ein solcher war das Germanin (Bayer 205), ein noch größerer die Entdeckung der Sulfonamide (Domagk, TrÉfouel, Nitti, Bovet 1935).

Und dann begann während der Kriegsjahre mit der Entdeckung und Einführung des Penicillins durch Fleming und Florey die neue Ära der Antibiotika, welche eine große Zahl von weiteren Infektionskrankheiten der erfolgreichen Behandlung zugänglich machten und die gegenüber den früheren Mitteln eine außerordentlich viel geringere Organotropie, d. h. Giftigkeit für den Wirt, aufweisen.

Nur in diesem einen Punkte hat der Optimismus Ehrlichs nicht genügt, um die spätere Forschung richtig vorauszusehen. In einem 1910 gehaltenen Vortrag führte er aus: «Die Heilsera besitzen keine Verwandtschaft zu den Körpersubstanzen. Es ist also in diesem Falle die Organotropie auf Null herabgesetzt, die Parasitotropie absolut, und es stellen somit die Antikörper Zauberkugeln dar, die ihr Ziel von selbst aufsuchen. Daher die wunderbare spezifische Wirkung und daher der Vorzug, den die Serumtherapie und die aktive Immunisierung vor jeder Chemotherapie voraus hat. Bei der Chemotherapie können wir auf solche Erfolge nie rechnen ${ }^{1}$ und werden daher alle Kräfte daran setzen müssen, möglichst scharf zu zielen, damit die Parasiten möglichst voll, der Körper möglichst wenig getroffen wird.»

Diesem letzteren Ziel ist die moderne Chemotherapie wenigstens bei einigen Antibiotika sehr nahe gekommen. Auch sie bewegt sich aber auf den Pfaden, die Paul Ehrlich, seiner Zeit weit vorauseilend, zu Beginn dieses Jahrhunderts geebnet und erstmals begangen hat.

Die Ansicht Ehrlichs, daß ein chemotherapeutisch wirksamer Stoff eine haptophore und eine toxophore Gruppe enthalten müsse - im Salvarsan

${ }^{1}$ In einem späteren Vortrag (1912) drückte er sich allerdings etwas optimistischer aus und meinte, «für ganz ausgeschlossen halte ich dies nicht». 
wurden der ortho-Aminophenolgruppe haptophore, dem Arsen toxophore Eigenschaften zugesprochen -, hat vielfache Kritik erfahren. Manche Forscher, wie z. B. Meyer und Overton, glaubten der Verteilung eines Stoffes zwischen wässeriger und lipoider Phase im Organismus eine große Bedeutung für dessen Wirksamkeit zuschreiben zu müssen. Wenn auch die Löslichkeitsverhältnisse der Pharmaka selbstverständlich von gewissem Einfluß sind, so kann doch auf Grund solcher Vorstellungen die große Konstitutionsspezifität der meisten Chemotherapeutika keine Erklärung finden. Auch die Wirkung der Antagonisten zahlreicher Heilmittel, z. B. der Sulfonamide als Antagonisten des Vitamins $p$-Aminobenzoesäure bzw. der Folsäure, kann nur - wenn überhaupt - auf konstitutionschemischer Grundlage verstanden werden. Die neueren Forschungen über Antibiotika, Chemotherapeutika und Vitamine haben aber zu der Erkenntnis geführt, daß die Wirkung solcher Stoffe auf Mikroorganismen oft nicht eine direkte, sondern indirekte sein mag, indem sie bestimmte Stoffe blockieren oder ausschalten, welche für die Mikroorganismen vitale Bedeutung besitzen und damit indirekt diese Mikroorganismen vernichten helfen. Daß der primäre Angriffspunkt mancher Pharmaka Fermente sind, konnte mehrfach sehr wahrscheinlich gemacht werden.

Paul Ehrlich hat fast auf allen Gebieten, auf denen er sich betätigte, Neuland betreten und grundlegende Entdeckungen gemacht, die vorher völlig unbekannt gewesen waren. Dieses Neuartige, oft der Zeit Vorauseilende, das häufig mit phantasievollen Bildern geschmückt und mit Leidenschaft vertreten wurde, forderte oft den Widerspruch und die Kritik von Fachkollegen heraus. Ehrlich hat viele wissenschaftliche Gegner gehabt und hat sich mit ihnen leidenschaftlich auseinandergesetzt, so z. B. mit МетsснNIKOFF und BoRDET. Die weitere Entwicklung der Forschung hat ihm meistens Recht gegeben. Wissenschaftliche Kritik hat ihn nicht gekränkt, sie war ihm Anregung zu neuen experimentellen Versuchen. Mancher glückliche Gedanke und manche geniale Eingebung blieben aber unverwirklicht, weil andere Arbeiten vorgingen. So hat er sich dem Sprechenden gegenüber schon im Jahre 1913 einmal geäußert, man müßte versuchen, eine Krankheit durch die Affizierung einer anderen zu heilen, wobei er ausdrücklich Malaria vorschlug. Das war vier Jahre bevor WAgner-JAuregg die Behandlung der Lues durch Malaria mit Erfolg anwandte.

An einen Freund in New York schrieb Ehrlich einmal, daß er eine besondere Fähigkeit besitze, alle seine Ideen und Probleme, die ihn beschäftigten. in seinem Geiste bildhaft zu sehen: die chemischen Formeln, die 
Seitenketten, die Rezeptoren, die Komplemente usw. ${ }^{2}$ Mit suggestiver Beredsamkeit suchte er diese Bilder und Vorstellungen anderen verständlich zu machen und durch Zeichnungen zu erläutern, die, wenn ein Schreibblock nicht zur Hand war, auf einer Schranktüre oder den Manschetten seiner Hemdärmel entworfen wurden. Fühlte er sich verstanden, so erfüllte ihn dies mit großer Genugtuung. In solchen Stunden schienen Außenwelt und Tagesfragen für ihn nicht zu existieren, zu denen er auch sonst nur zögernd und zurückhaltend Stellung bezog.

Paul Ehrlich hat alle Ehrungen erfahren, die die Welt einem großen Gelehrten zuteil werden lassen kann. Sein eigenes Vaterland und das Ausland haben mit Dankbezeugungen aller Art nicht gekargt. Sie haben den einfachen, bescheidenen Mann nicht geändert. Nach wie vor lebte er allein seiner Arbeit. Eine Eigenschaft Paul Ehrlichs haben nur diejenigen erfahren, die ihn näher kennenlernen durften. Das ist seine große menschliche Güte; diese ist vielen Kranken, vielen Hilfesuchenden und auch manchen seiner engeren Mitarbeiter zuteil geworden.

Meine Zusammenarbeit mit Paul Ehrlich dauerte nur drei Jahre; es waren seine letzten Lebensjahre. Jene Zeit gehört zu den glücklichsten Abschnitten meines Lebens. Wir pflegten täglich oft stundenlange Aussprachen über wissenschaftliche Probleme. Ehrlichs Sekretärin, Fräulein MARQUARDT, schreibt in ihrem Buch über Paul Ehrlich, daß er und ich schnell Freunde wurden. Ich würde eher sagen, daß er mir, der ich damals noch sehr jung war, ein väterlicher Freund gewesen ist, dem ich mich zutiefst verpflichtet fühle.

In der Geschichte der Naturwissenschaften und der Medizin wird der Name Paul Ehrlichs immer in unvergänglichen Lettern stehenbleiben. Mit seiner reichen Phantasie und seinem scharfen, kritischen Verstand vereinigte er Gaben, mit denen er Wege öffnen und Probleme erfolgreich angehen konnte, die vorher völlig unberührt gewesen waren. Er hat nach dem Rat gehandelt, den ein anderer Heroe der Naturwissenschaften, August KEкuLÉ, einmal den Forschern gegeben hat:

«Lernen wir träumen, dann finden wir vielleicht die Wahrheit:

Und wer nicht denkt,

Dem wird sie geschenkt,

Er hat sie ohne Sorgen.

Aber hüten wir uns, unsere Träume anderen mitzuteilen,

Ehe sie durch den wachenden Verstand geprüft worden sind.»

${ }^{2}$ Zitiert nach Martha Marquard, Paul Ehrlich, London 1949. 